\title{
Spontaneous Decomposition of Fluorinated Phosphorene and Its Stable Structure
}

\author{
Artem V. Kuklin, ${ }^{*} \dagger, \ddagger$ Glib V. Baryshnikov $,{ }^{\dagger}, \S$ and Hans Ågren ${ }^{*}, \|, \dagger$ \\ ${ }^{\dagger}$ Division of Theoretical Chemistry and Biology, School of Engineering Sciences in Chemistry, Biotechnology and Health, \\ KTH Royal Institute of Technology, 10691, Stockholm, Sweden \\ ${ }^{\ddagger}$ Division of Theoretical Physics and Wave Phenomena, Siberian Federal University, 79 Svobodniy av., Krasnoyarsk \\ 660041, Russia \\ ${ }^{\S}$ Department of Chemistry and Nanomaterials Science, Bohdan Khmelnytsky National University, 18031, Cherkasy, \\ Ukraine \\ ${ }^{\dagger}$ College of Chemistry and Chemical Engineering, Henan University, Kaifeng, Henan 475004, P.R. China
}

The Vienna Ab Initio Simulation Package (VASP) ${ }^{1,2}$ was utilized to simulate bare and fluorinated phosphorene nanostructures in the framework of Density Functional Theory (DFT) and plane-wave (PW) basis set. The projector augmented wave (PAW) $)^{3}$ method with a cutoff energy of $400 \mathrm{eV}$ and generalized gradient approximation (GGA) of Perdew-Burke-Ernzerhof (PBE) ${ }^{4}$ exchange functional were employed within periodic boundary condition (PBC). The hybrid density functional proposed by Heyd-Scuseria-Ernzerhof (HSE06) ${ }^{5}$ was adopted for band structure calculations. Vander-Waals-London dispersion interactions were corrected by D3 Grimme method. ${ }^{6}$ The reciprocal space in structural optimizations and band structure calculations was sampled by $12 \times 12 \times 1$ and 10 $\times 8 \times 1 k$-points generated using $\Gamma$-centred Monkhorst-Pack scheme ${ }^{7}$ for monolayer $\mathrm{PF}_{2}$ and $\mathrm{PF}$, respectively. A vacuum region of $>12 \AA$ set to avoid artificial interaction between neighboring images. The convergence for the force and electronic minimizations were set no less than $10^{-3} \mathrm{eV} / \AA$ and $10^{-}$ ${ }^{5} \mathrm{eV}$, respectively. Random cluster simulation using the CALYPSO code ${ }^{8}$ were performed with population size equal to 50. VESTA software ${ }^{9}$ was used to produce some figures. The thermal stability of fluorinated phosphorene nanostructures was evaluated using ab-initio molecular dynamics (AIMD) approach $^{10,11}$ within canonical (NVT) ensemble ${ }^{12}$ and Nosé-Hoover thermostat. ${ }^{13,14}$ Time step of 1 fs at the temperature of $350 \mathrm{~K}$ was conditioned during AIMD simulation. The size of supercells was varied from $4 \times 4 \times 1$ in tetragonal structures to $5 \times 5 \times 1$ in the hexagonal one. Molecular electrostatic potential maps were calculated using GAUSSIAN16 ${ }^{15}$ within B3LYP/6-31++G(d,p $)^{16-19}$ basis set. 


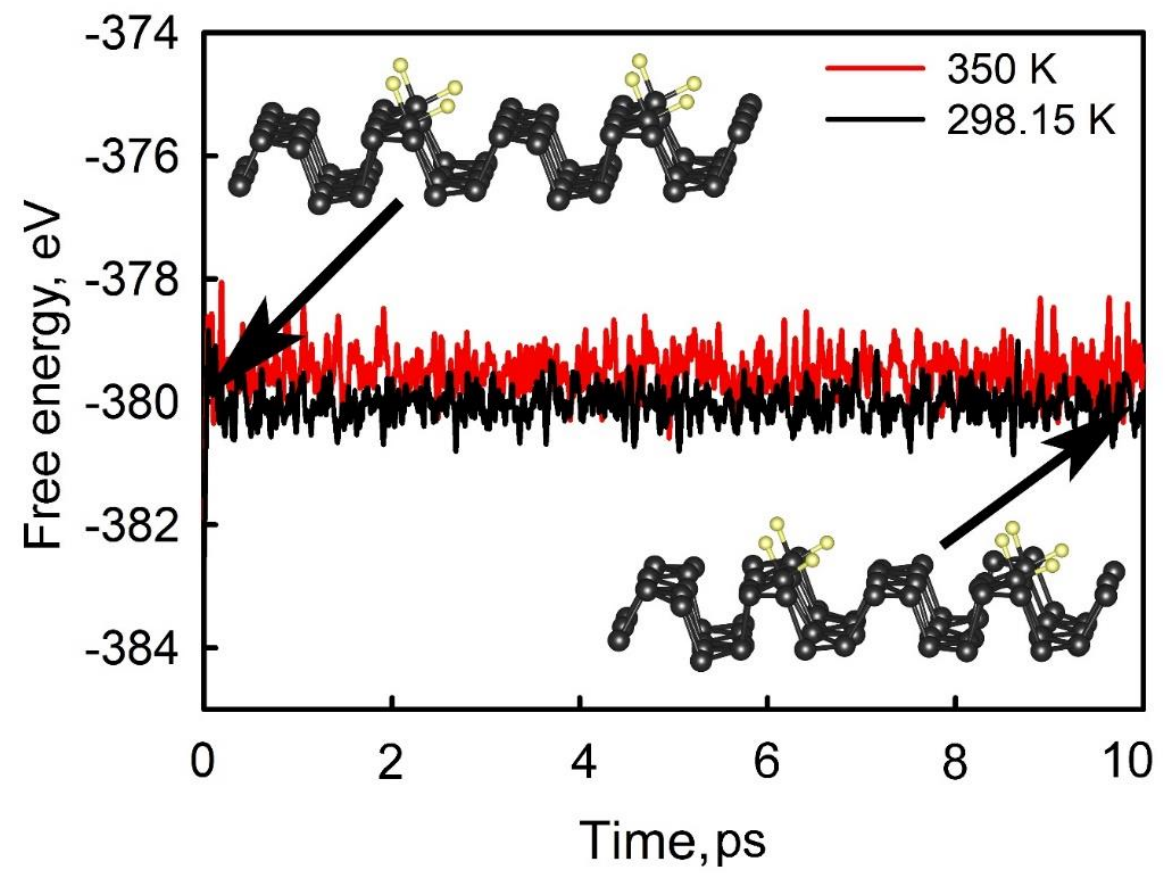

Figure S1. Evolution of free energy of the fluorinated phosphorene $\mathrm{P}_{16} \mathrm{~F}_{2}$ structure during the AIMD simulation at 298.15 and $350 \mathrm{~K}(10 \mathrm{ps})$ within the NVT ensemble. The $2 \times 2 \times 1$ supercell contained 72 atoms in total was used as the initial structure.
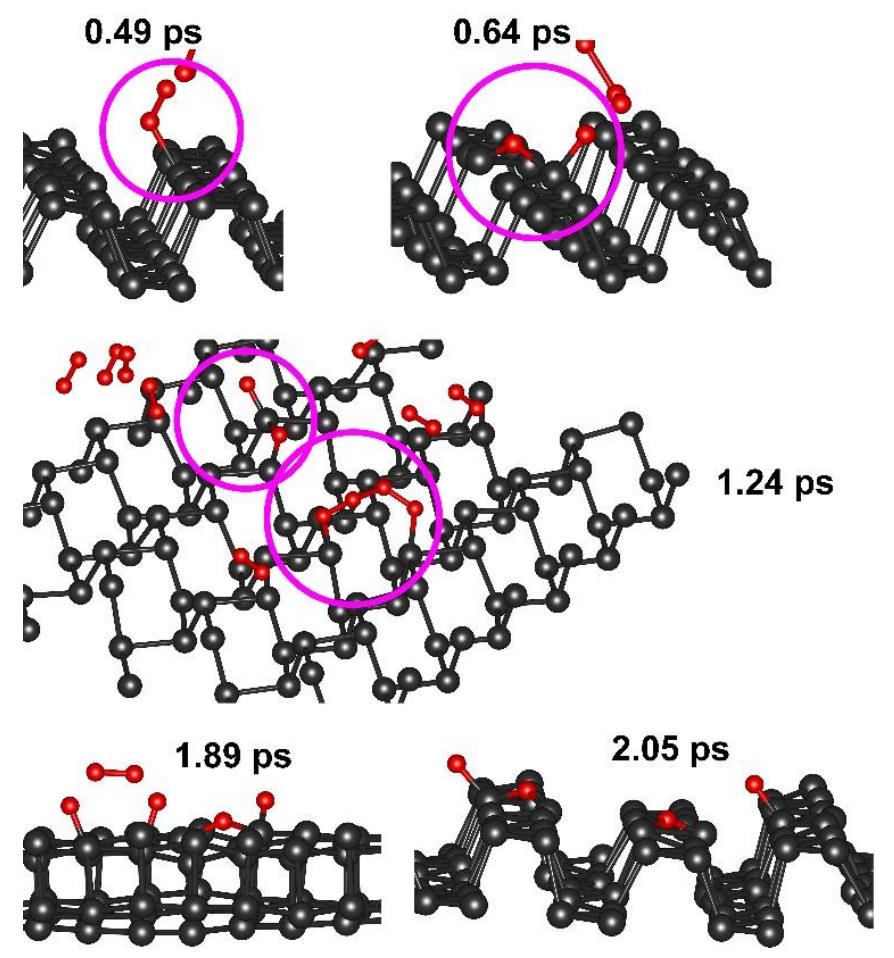

Figure S2. AIMD snapshots of the configurations demonstrating interaction of oxygen with pristine phosphorene. The AIMD simulation was carried out at $298.15 \mathrm{~K}$ within the NVT ensemble and time step of $1 \mathrm{fs}$ during $10 \mathrm{ps}$. The supercell contained $64 \mathrm{P}$ atoms was used as the initial structure. 


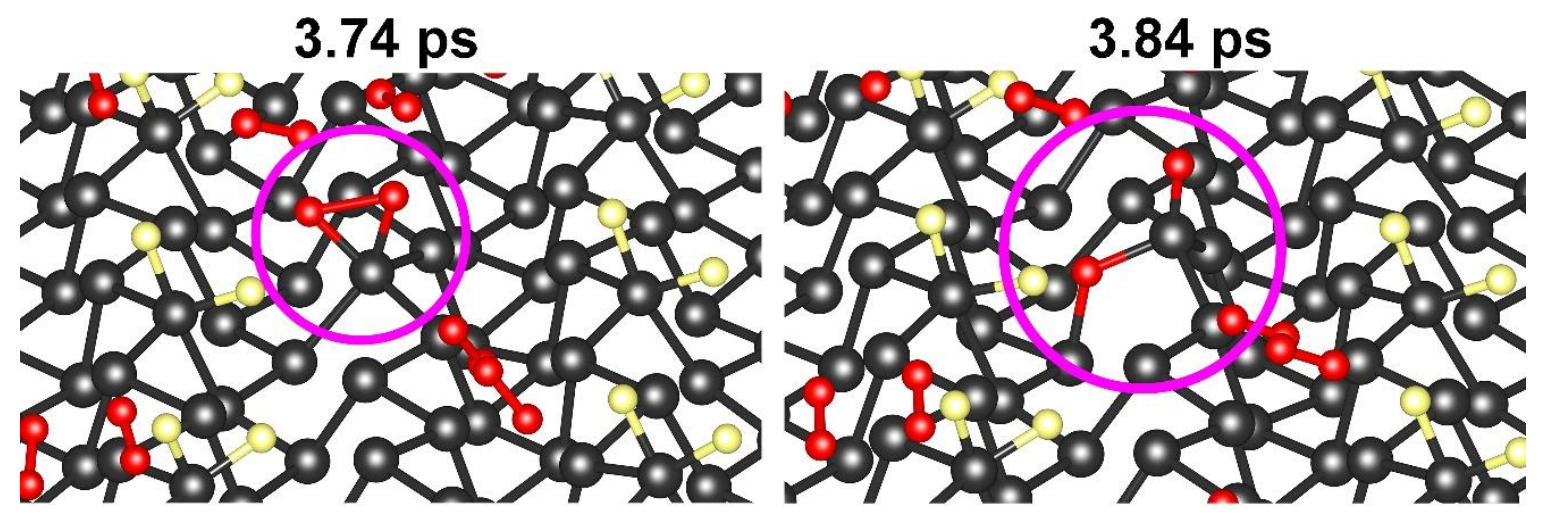

Figure S3. AIMD snapshots of the configurations demonstrating interaction of oxygen with fluorinated phosphorene. The AIMD simulation was carried out at $298.15 \mathrm{~K}$ within the NVT ensemble and time step of $1 \mathrm{fs}$ during $10 \mathrm{ps}$. The supercell contained $64 \mathrm{P}$ and $8 \mathrm{~F}$ atoms was used as the initial structure.

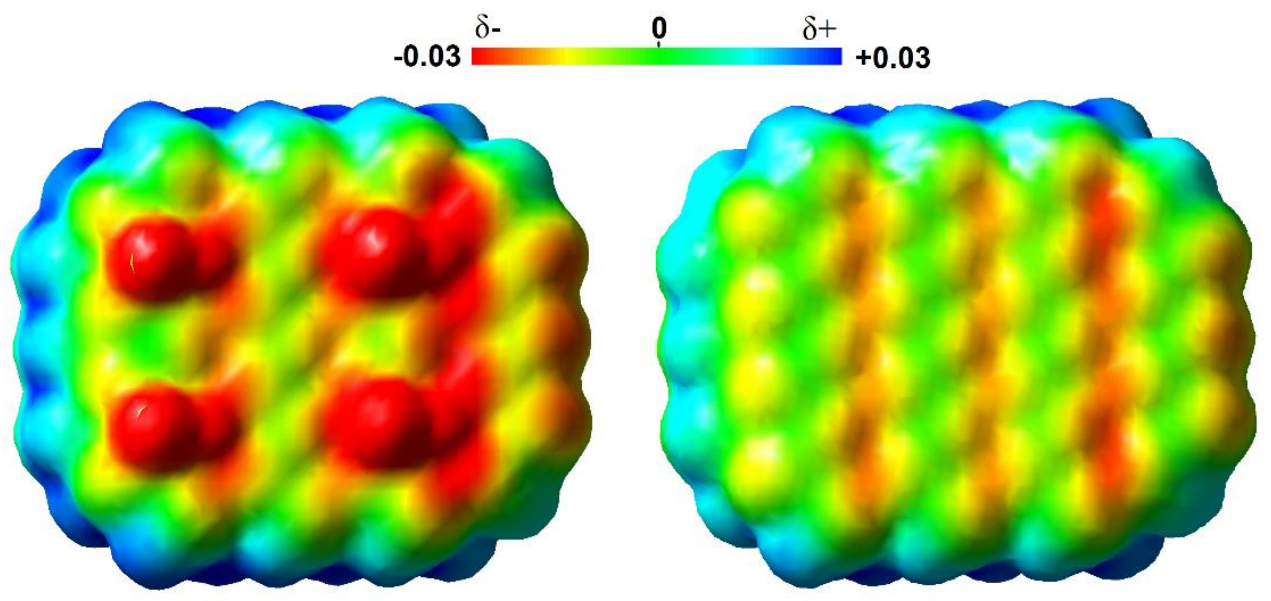

Figure S4. Molecular electrostatic potential maps generated with B3LYP/6-31++G(d,p) level electron density for the fluorinated (left) and bare (right) phosphorene fragments. Electrostatic potentials are mapped with isosurface set to 0.0004 a.u. The red surface corresponds to a negative region of electrostatic potential (-0.03 a.u.), whereas the blue color corresponds to the region where potential is positive (+0.03 a.u.).

\section{References}

(1) Kresse, G.; Hafner, J. Norm-Conserving and Ultrasoft Pseudopotentials for First-Row and Transition Elements. J. Phys. Condens. Matter 1994, 6, 8245-8257.

(2) Kresse, G.; Furthmüller, J. Efficient Iterative Schemes for Ab Initio Total-Energy Calculations Using a Plane-Wave Basis Set. Phys. Rev. B 1996, 54, 11169-11186. 
(3) Blöchl, P. E. Projector Augmented-Wave Method. Phys. Rev. B 1994, 50, 17953-17979.

(4) Perdew, J. P.; Burke, K.; Ernzerhof, M. Generalized Gradient Approximation Made Simple. Phys. Rev. Lett. 1996, 77, 3865-3868.

(5) Heyd, J.; Scuseria, G. E.; Ernzerhof, M. Hybrid Functionals Based on a Screened Coulomb Potential. J. Chem. Phys. 2003, 118, 8207.

(6) Grimme, S. Semiempirical GGA-Type Density Functional Constructed with a Long-Range Dispersion Correction. J. Comput. Chem. 2006, 27, 1787-1799.

(7) Monkhorst, H.; Pack, J. Special Points for Brillouin Zone Integrations. Phys. Rev. B 1976, 13, 5188-5192.

(8) Wang, Y.; Lv, J.; Zhu, L.; Ma, Y. CALYPSO: A Method for Crystal Structure Prediction. Comput. Phys. Commun. 2012, 183, 2063-2070.

(9) Momma, K.; Izumi, F. VESTA 3 for Three-Dimensional Visualization of Crystal, Volumetric and Morphology Data. J. Appl. Crystallogr. 2011, 44, 1272-1276.

(10) Kresse, G.; Hafner, J. Ab Initio Molecular Dynamics for Liquid Metals. Phys. Rev. B 1993, $47,558-561$.

(11) Kresse, G.; Hafner, J. Ab Initio Molecular-Dynamics Simulation of the Liquid-MetalAmorphous-Semiconductor Transition in Germanium. Phys. Rev. B 1994, 49, 14251-14269.

(12) Martyna, G. J.; Klein, M. L.; Tuckerman, M. Nosé-Hoover Chains: The Canonical Ensemble via Continuous Dynamics. J. Chem. Phys. 1992, 97, 2635-2643.

(13) Nosé, S. A Unified Formulation of the Constant Temperature Molecular Dynamics Methods. J. Chem. Phys. 1984, 81, 511-519.

(14) Evans, D. J.; Holian, B. L. The Nose-Hoover Thermostat. J. Chem. Phys. 1985, 83, 40694074.

(15) Frisch, M. J.; Trucks, G. W.; Schlegel, H. B.; Scuseria, G. E.; Robb, M. A.; Cheeseman, J. R.; Scalmani, G.; Barone, V.; Petersson, G. A.; Nakatsuji, H. . et al. Gaussian 16, Revision C.01; Gaussian, Inc.: Wallingford CT, 2016.

(16) Frisch, M. J.; Pople, J. A.; Binkley, J. S. Self-consistent Molecular Orbital Methods 25. Supplementary Functions for Gaussian Basis Sets. J. Chem. Phys. 1984, 80, 3265-3269.

(17) Krishnan, R.; Binkley, J. S.; Seeger, R.; Pople, J. A. Self-consistent Molecular Orbital Methods. XX. A Basis Set for Correlated Wave Functions. J. Chem. Phys. 1980, 72, 650654.

(18) Clark, T.; Chandrasekhar, J.; Spitznagel, G. W.; Schleyer, P. V. R. Efficient Diffuse Function-Augmented Basis Sets for Anion Calculations. III. The 3-21+G Basis Set for FirstRow Elements, Li-F. J. Comput. Chem. 1983, 4, 294-301.

(19) Becke, A. D. Density-functional Thermochemistry. III. The Role of Exact Exchange. J. Chem. Phys. 1993, 98, 5648-5652. 